\title{
Contrast-enhanced ultrasound to visualize hemodynamic changes after rodent spinal cord injury
}

\author{
Zin Z. Khaing, PhD, ${ }^{1}$ Lindsay N. Cates, BS, ${ }^{1}$ Dane M. DeWees, BS, ${ }^{1}$ Alexander Hannah, $\mathrm{PhD},{ }^{2}$ \\ Pierre Mourad, PhD, ${ }^{1}$ Matthew Bruce, PhD, ${ }^{2}$ and Christoph P. Hofstetter, MD, PhD'1 \\ 'Department of Neurological Surgery and ${ }^{2}$ Applied Physics Laboratory, Center for Industrial and Medical Ultrasound, The \\ University of Washington, Seattle, Washington
}

\begin{abstract}
OBJECTIVE Traumatic spinal cord injury (tSCl) causes an almost complete loss of blood flow at the site of injury (primary injury) as well as significant hypoperfusion in the penumbra of the injury. Hypoperfusion in the penumbra progresses after injury to the spinal cord and is likely to be a major contributor to progressive cell death of spinal cord tissue that was initially viable (secondary injury). Neuroprotective treatment strategies seek to limit secondary injury. Clinical monitoring of the temporal and spatial patterns of blood flow within the contused spinal cord is currently not feasible. The purpose of the current study was to determine whether ultrafast contrast-enhanced ultrasound (CEUS) Doppler allows for detection of local hemodynamic changes within an injured rodent spinal cord in real time.
\end{abstract}

METHODS A novel ultrafast CEUS Doppler technique was developed utilizing a research ultrasound platform combined with a 15-MHz linear array transducer. Ultrafast plane-wave acquisitions enabled the separation of higher-velocity blood flow in macrocirculation from low-velocity flow within the microcirculation (tissue perfusion). An FDA-approved contrast agent (microbubbles) was used for visualization of local blood flow in real time. CEUS Doppler acquisition protocols were developed to characterize tissue perfusion both during contrast inflow and during the steady-state plateau. A compression injury of the thoracic spinal cord of adult rats was induced using iris forceps.

RESULTS High-frequency ultrasound enabled visualization of spinal cord vessels such as anterior spinal arteries as well as central arteries (mean diameter [ \pm SEM] $145.8 \pm 10.0 \mu \mathrm{m} ; 76.2 \pm 4.5 \mu \mathrm{m}$, respectively). In the intact spinal cord, ultrafast CEUS Doppler confirmed higher perfusion of the gray matter compared to white matter. Immediately after compression injury of the thoracic rodent spinal cord, spinal cord vessels were disrupted in an area of $1.93 \pm 1.14 \mathrm{~mm}^{2}$. Ultrafast CEUS Doppler revealed a topographical map of local tissue hypoperfusion with remarkable spatial resolution. Critical loss of perfusion, defined as less than $40 \%$ perfusion compared to the surrounding spared tissue, was seen within an area of $2.21 \pm 0.6 \mathrm{~mm}^{2}$.

CONCLUSIONS In our current report, we introduce ultrafast CEUS Doppler for monitoring of spinal vascular structure and function in real time. Development and clinical implementation of this type of imaging could have a significant impact on the care of patients with $\mathrm{tSCl}$.

https://thejns.org/doi/abs/10.3171/2018.1.SPINE171202

KEYWORDS spinal cord injury; rodent injury models; contrast-enhanced ultrasound imaging; intraoperative ultrasound

$\mathrm{T}$ RaUmatic spinal cord injury (tSCI) evolves in 2 phases: the primary injury is characterized by direct mechanical tissue destruction and is followed by secondary injury of tissue surrounding the injury center. ${ }^{6}$ Ischemia is thought to be a major contributor to secondary injury. ${ }^{25}$ Traumatic SCI causes an almost complete loss of blood flow at the injury center and significant hy- poperfusion of the penumbral zone with progressive cell death over time..$^{10,16,25}$ Significant research efforts are ongoing to develop experimental neuroprotective therapies targeting this "rescue-able" penumbral zone. In brain trauma, intensive care interventions aim to continuously adjust arterial blood pressure and intracranial pressure to optimize tissue perfusion. This approach has resulted in

ABBREVIATIONS CEUS = contrast-enhanced ultrasound; SCI = spinal cord injury; SEM = standard error of the mean; $\mathrm{tSCl}=$ traumatic SCI.

SUBMITTED October 31, 2017. ACCEPTED January 11, 2018.

INCLUDE WHEN CITING Published online June 15, 2018; DOI: 10.3171/2018.1.SPINE171202. 
significant reduction of mortality and morbidity. ${ }^{24,27}$ While current spine trauma guidelines recommend maintenance of a mean arterial pressure greater than $85 \mathrm{~mm} \mathrm{Hg},{ }^{17}$ intensive management of local tissue perfusion of the contused spinal cord is currently not feasible due to a lack of appropriate biomarkers.

Ultrasound imaging holds great promise for intraoperative neurosurgical applications. ${ }^{28}$ Ultrasound equipment is readily available in the operative suite, surgeons are well versed in its use, it is relatively inexpensive, and it does not expose patient or surgeon to potentially harmful radiation. Our group has developed a technique that combines contrast-enhanced ultrasound (CEUS) with ultrafast plane-wave acquisitions-referred to as ultrafast CEUS Doppler-and has applied this to in vivo spinal cord imaging. ${ }^{36-38}$ This technique allows for simultaneous visualization of high-velocity blood flow in the macrocirculation, which transports blood between organs, and low-velocity flow in the microcirculation, where capillary exchange of oxygen, electrolytes, and nutrients occurs. This novel technology has not been previously applied to visualize vasculature in the spinal cord. In the current study, we demonstrate that ultrafast CEUS Doppler can assess the integrity of spinal cord blood vessels and map hypoperfused spinal cord tissue in a rodent tSCI model. This technology has the potential to serve as a novel preclinical and clinical biomarker, detailing the hemodynamic changes within the spinal cord tissue in real time.

\section{Methods}

All materials used in the following experiments were obtained from Sigma-Aldrich unless otherwise noted.

\section{In Vitro Phantom Flow Studies}

An infusion pump (Chemyx N3000) was used to pump distilled water with Definity (Lantheus) microbubbles through a 2-mm dialysis tube, which was suspended in an acrylic box filled with water. The Vantage ultrasound research platform (Verasonics) was used to program single-angle plane-wave nonlinear Doppler sequences. A $15-\mathrm{MHz}$ linear array transducer (Vermon) was used and supported by a mounting arm (FISSO). Acquisitions were collected for both stationary and flowing microbubbles. Velocity measurements of flowing microbubbles were validated using spectral Doppler on a commercial ultrasound system (Epiq, Philips Medical Systems).

\section{Rodent Spinal Cord Injury Model}

Surgical procedures were performed according to approved institutional animal care and use committee (IACUC) protocol following all appropriate guidelines from the university's Animal Welfare Assurance (A346401) as well as the NIH Office of Laboratory Animal Welfare (OLAW). Female Sprague-Dawley rats $(n=5,250-$ $300 \mathrm{~g}$ at the time of experiment; Harlan Laboratories) were used. The rats were anesthetized using isoflurane (5\% for induction and $2.5 \%-3 \%$ for maintenance), and the skin overlying the T2-12 vertebrae was shaved, cleaned, and disinfected. A longitudinal incision was carried out overlying the T6-11 area using a no. 10 scalpel blade. Af- ter subperiosteal dissection of paraspinal muscles, a laminectomy was performed to expose the spinal cord from T6 to T10. A compression-type lesion was produced using a calibrated compression method (Graefe Iris forceps with 0.8 -mm tip for 30 seconds). ${ }^{2,26}$ The rats received lactated Ringer's solution during anesthesia (administered subcutaneously; $5 \mathrm{ml}$ for every 2 hours).

\section{Ultrasound Imaging With Contrast Agent and Image Acquisition}

Warm and de-gassed ultrasound gel was applied directly onto the exposed dorsal surface of the T6-10 spinal cord. For ultrasound imaging, the Vantage ultrasound research system (Verasonics) was used in combination with a $15-\mathrm{MHz}$ transducer (Vermon). For each CEUS, a bolus of $0.15 \mathrm{ml}$ of Definity (Lantheus) ultrasound contrast agent was injected intravenously, followed by a 0.2$\mathrm{ml}$ saline flush. Plane-wave nonlinear contrast-enhanced sagittal images were acquired over the entirety of the bolus administration for time-intensity analysis. ${ }^{38}$ For each acquisition in our study, video screen captures of the passage of the microbubble bolus were recorded (approximately 1 minute). Regions of interests were placed in the hypoechoic center of the injury, in tissue adjacent to the injury, and in intact tissue further from the injury center. The mean intensity of the microbubble signal was plotted over time to compare bolus kinetics between these 3 regions.

In addition, ultrafast plane-wave acquisitions combined with nonlinear pulsing sequences were acquired, enabling assessment of perfusion and larger vascular flow. ${ }^{36-38} \mathrm{~A}$ Doppler sequence of alternating amplitudes was transmitted and then summed, generating a nonlinear Doppler sequence where the stronger nonlinear signals from microbubbles are preserved and weaker nonlinear echoes from tissue are suppressed. ${ }^{37}$ Doppler processing of this sequence separated low-velocity stationary microbubbles in the microcirculation from higher-velocity microbubbles in larger vasculature. ${ }^{37}$ In the current study we refer to the low-velocity blood flow in the microcirculation as tissue perfusion. Acquisitions were obtained before the injury and 15 minutes after injury in each animal. All spinal CEUS imaging was done as a nonsurvival procedure (see below). The Doppler flow images of the larger vasculature were used to analyze vessel diameter of the anterior spinal and central arteries. For quantification of vessel diameter, 4 random areas of the anterior spinal and central arteries were selected per animal. The vessel diameter was determined using a calibrated length measurement probe in Adobe Photoshop CS5.

Perfusion images of each animal were co-registered to the center of the hypoechoic lesion site. The mean perfusion intensity for each pixel was then calculated from the co-registered images. The inversion of the mean perfusion map was used to create a heat map to estimate the overall extent of injury for the group of rats. The white of the heat map corresponds to complete lack of microbubble signal found at the site of injury and labeled as $100 \%$ injury, with yellow and orange corresponding to graded deficits in microbubble signal relative to peak microbubble signal outside the zone of injury, labeled as $0 \%$ injury. In addi- 
tion, the contour of $40 \%$ of the peak perfusion signal in intact spinal cord tissue was used to calculate the area of primary injury for each rat.

\section{Histology}

At the end of the experiment, animals were euthanized using an overdose of Beuthanesia-D (Schering-Plough Animal Health). Transcardiac perfusion was performed using ice-cold phosphate-buffered saline ( $\mathrm{pH} 7.4$, approximately $200 \mathrm{ml}$ ) followed by cold $4 \%$ paraformaldehyde (approximately $300 \mathrm{ml}$ ). Entire spinal columns were removed, postfixed in $4 \%$ paraformaldehyde solution overnight at $4{ }^{\circ} \mathrm{C}$, and treated with $30 \%$ sucrose solution with $0.01 \%$ sodium azide prior to freezing. Seven-millimeter segments centered over T6-10 were dissected and frozen, and $20-\mu \mathrm{m}$-thick longitudinal sections were obtained using a cryostat (Leica, CM1850), thaw mounted onto gelatin-coated glass slides, and stored at $-80^{\circ} \mathrm{C}$. Standard hematoxylin and eosin staining was performed on longitudinal sections. Photomicrographs were taken using a Zeiss Primo Star microscope with a color camera (Axiocam ERc 5s) attached. Images were processed using either Adobe Photoshop CS5.1 or Adobe Illustrator CS6.

\section{Statistical Analysis}

Continuous variables are shown as means \pm standard error of the mean (SEM). Statistical calculations were carried out using SPSS 24 for Mac. Acquired ultrasound data were analyzed using Ultra-Extend software (Toshiba). The software generated a perfusion-reperfusion curve (from baseline to 60 seconds) for each region of interest (ROI) per acquisition, and calculated the area under the curve (AUC) directly correlated to spinal cord blood flow.

\section{Results}

\section{CEUS-Based Detection of Blood Flow}

Ultrafast CEUS Doppler In Vitro Separation of Stationary and Flowing Microbubbles

The ability of ultrafast CEUS Doppler to distinguish between microbubbles suspended in stationary and moving fluid was tested using an in vitro flow phantom system. This experiment demonstrated that ultrafast CEUS Doppler reliably detects and separates stationary from moving microbubbles (Fig. 1). Figure 1c confirmed the absence of moving bubbles in the stationary fluid, and the presence of flow (Fig. 1d) in moving fluid in a flow phantom. Peak velocity flow was estimated at $3 \mathrm{~cm} / \mathrm{sec}$, with a parabolic velocity profile (Fig. 1f).

\section{Ultrafast CEUS Doppler In Vivo Detection of Larger Vasculature}

CEUS visualization of spinal cord blood vessels was carried out in the midsagittal plane of the intact rodent thoracic spinal cord (Fig. 2a and d, Video 1).

VIDEO 1. Recording of contrast inflow of a sagittal section of the intact thoracic rodent spinal cord. Copyright University of Washington. Published with permission. Click here to view.

Ultrafast CEUS Doppler revealed the vascular anatomy of the intact spinal cord with excellent spatial resolution. Analysis of the anterior spinal artery revealed a mean
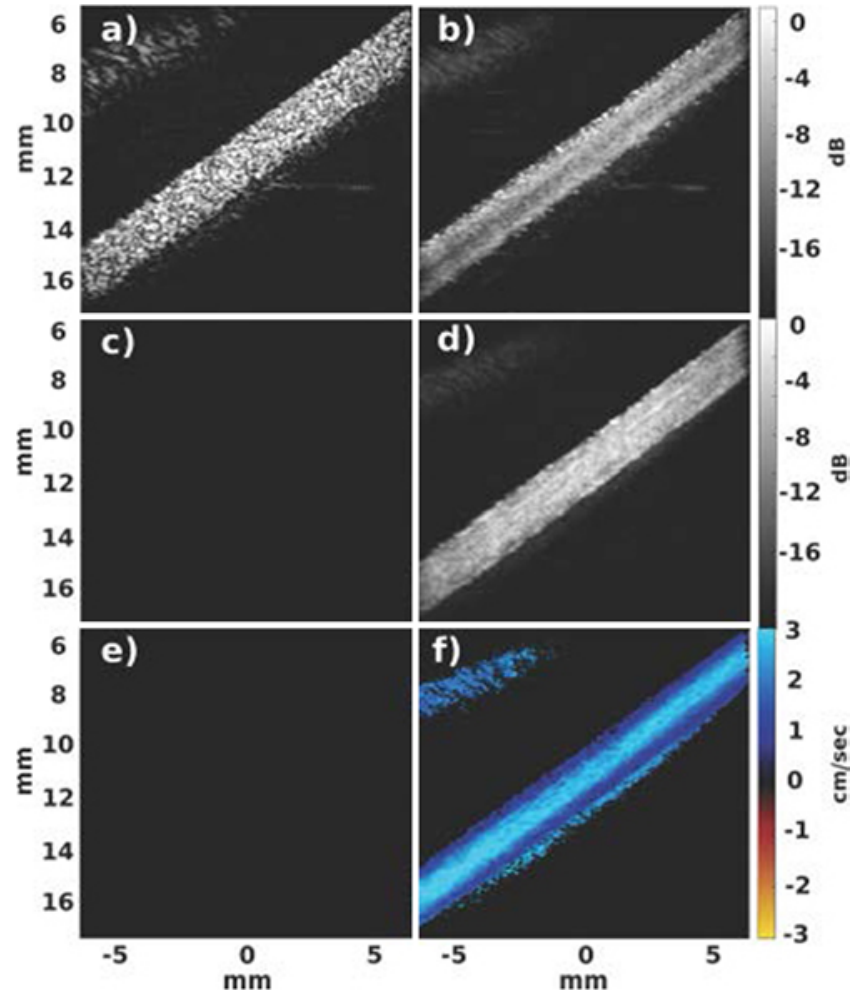

FIG. 1. Illustration of ultrafast CEUS Doppler's ability to distinguish between stationary (a, c, and e) and flowing (b, d, and g) microbubbles, as well as estimate the velocity of higher-velocity microbubbles (f). a: Stationary microbubbles are detected using ultrafast CEUS Doppler. b: Detection of flowing microbubbles. c and e: Doppler filtering removing the nonlinear Doppler signals from stationary microbubbles. d: Doppler filtering preserving nonlinear Doppler signals from moving microbubbles. f: Velocity estimates showing a parabola-like flow profile having a peak central velocity of $3 \mathrm{~cm} / \mathrm{sec}$. Figure is available in color online only.

diameter of $145.8 \pm 10.0 \mu \mathrm{m}$. Central arteries branching off the anterior spinal artery were measured as $76.2 \pm 4.5$ $\mu \mathrm{m}$ in mean diameter. We also routinely visualized the posterior spinal arterial network (Fig. 2b and e). Velocity measurements of vascular flow revealed an average blood flow velocity of $4-6 \mathrm{~cm} / \mathrm{sec}$ in larger spinal cord vessels (> $50 \mu \mathrm{m}$ in diameter). A compression injury of the thoracic spinal cord resulted in a complete disruption of microvasculature in the injury center (Fig. 2, Video 2).

VIDEO 2. Recording of contrast inflow of a sagittal section of the acutely injured thoracic rodent spinal cord. Copyright University of Washington. Published with permission. Click here to view.

Thus, disruption of microvasculature was measured in an average area of $1.93 \pm 1.14 \mathrm{~mm}^{2}$ approximately 15 minutes after injury. Hemorrhage and edema at the injury center resulted in mass effect onto surrounding spinal cord vasculature.

\section{CEUS Estimates Local Tissue Perfusion}

Ultrafast CEUS Doppler imaging detected local tissue perfusion in intact and injured thoracic spinal cord (Fig. $2 \mathrm{c}$ and $\mathrm{f}$ and Fig. 3 left). In the midsagittal plane of the 


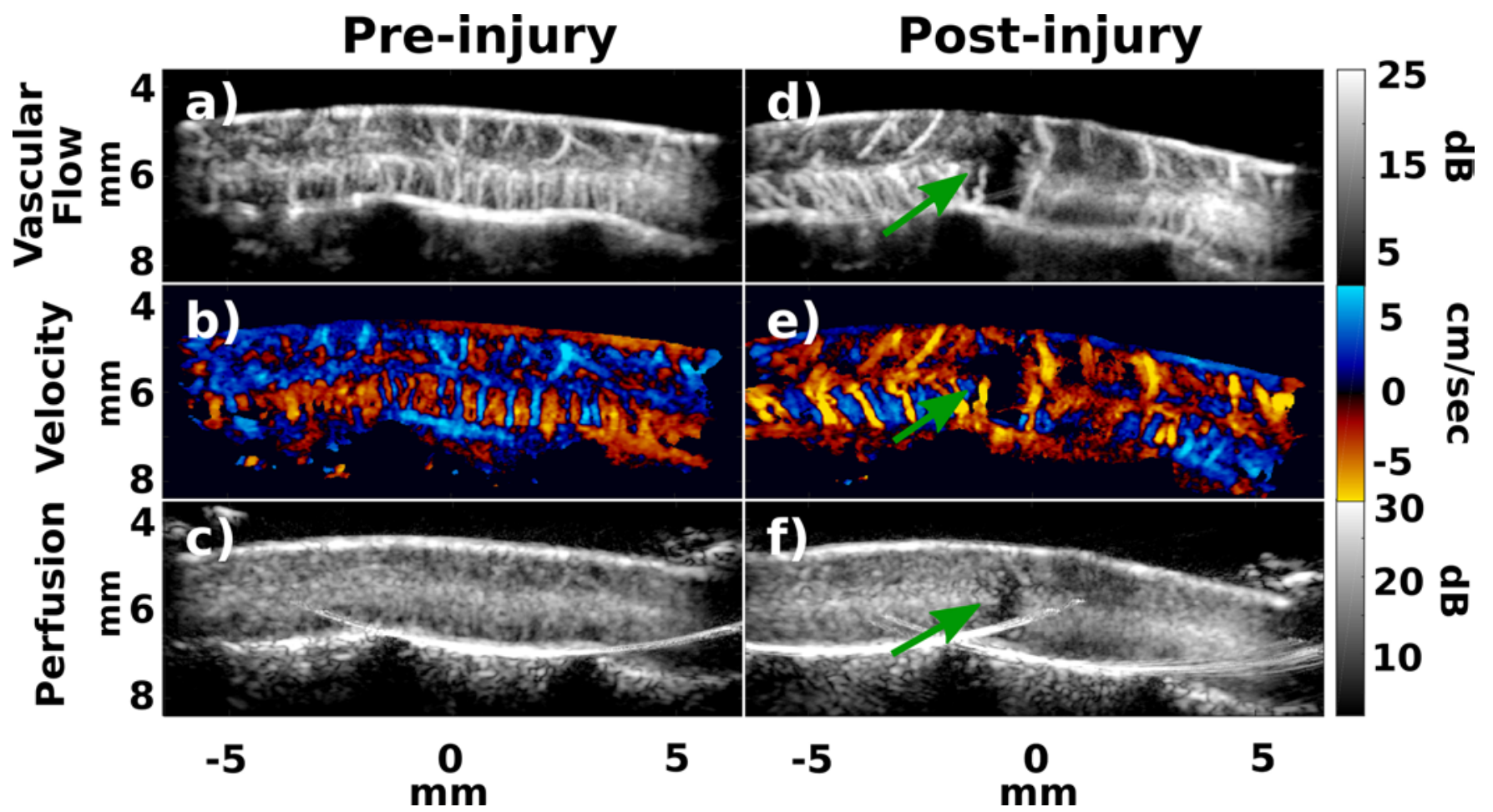

FIG. 2. Ultrafast CEUS Doppler imaging of spinal vasculature and blood flow in macro- and microcirculations before (a-c) and after $(d-f)$ injury in a rodent spinal cord. Panels a and $d$ show higher-velocity microbubble flow in the larger vasculature before and after $\mathrm{tSCl}$, respectively. Panels $\mathbf{b}$ and e show estimates of microbubble velocity in the larger vasculature before and after injury, respectively. Panels $\mathbf{c}$ and $\mathbf{f}$ show the lower-velocity microbubbles in the microcirculation (i.e., perfusion) before and after injury, respectively. $\mathrm{dB}=$ decibel—ratio of Doppler power signals represented in log scale to the lowest signal displayed in black.

intact spinal cord, greater perfusion signals were visualized in the central region of the spinal cord, likely corresponding to the differences in vascular density between gray and white matter (Fig. 2c). Following a compression injury, a dramatic loss of tissue perfusion was detected in the injury center (Figs. 2 and 3).

We then further analyzed perfusion characteristics of the injury center and penumbra during the inflow of the microbubble bolus. Figure 3 compares the bolus kinetics in the intact, injured, and penumbral region of an acutely injured rodent spinal cord. Contrast inflow to the adjacent penumbral area was significantly reduced and delayed. A range of a 1- to 2-second delay in peak microbubble infusion was recorded in hypoperfused lesion penumbra compared to the intact spinal cord. A near-complete absence of microbubble perfusion signal was observed at the site of injury epicenter.

We then analyzed perfusion signal during the steadystate plateau of contrast agent in the lesion center and hypoperfused penumbra (Fig. 4). Utilizing a $40 \%$ of peak perfusion signal contour of the primary injury, compression injuries of the thoracic spinal cord resulted in a mean area of $2.21 \pm 0.6 \mathrm{~mm}^{2}$ of critically low tissue perfusion. Co-registration of individual perfusion studies allowed for calculation of a mean perfusion heat map. Interestingly, the area of the injury with less than $40 \%$ tissue perfusion compared to surrounding healthy tissue showed close re- semblance to the necrotic injury core seen on an $\mathrm{H}$ \& Estained sagittal section.

\section{Discussion}

In the current study, we introduce ultrafast CEUS Doppler for structural and functional characterization of spinal cord vasculature. Following tSCI, CEUS can quantify the extent of disrupted microvasculature and produce a topographical map of tissue hypoperfusion with a spatial resolution to resolve perfusion differences in and surrounding the site of injury.

Traumatic SCI causes an almost complete loss of blood flow in the injury center and significant hypoperfusion in the penumbral zone with progressive cell death over time. ${ }^{10,16,25}$ Ischemia is thought to be a major contributor to secondary injury. ${ }^{25} \mathrm{~A}$ recent clinical study demonstrated that both clinical motor response and amplitude of motor evoked potentials correlate with spinal perfusion pressure in patients with acute tSCI. ${ }^{40}$ Moreover, hypoperfusion exacerbates secondary injury by progressive cell death over time. ${ }^{10,16,25}$ Indeed, two routinely used clinical treatment strategies aim to improve the local tissue perfusion of the contused spinal cord. First, surgical decompression of the spinal cord is recommended within 24 hours after injury, as it may improve functional outcome. ${ }^{5,13,15,41,42}$ Second, trauma guidelines recommend maintenance of the mean arterial blood pressure at $85-90 \mathrm{~mm} \mathrm{Hg}$ for the first 7 

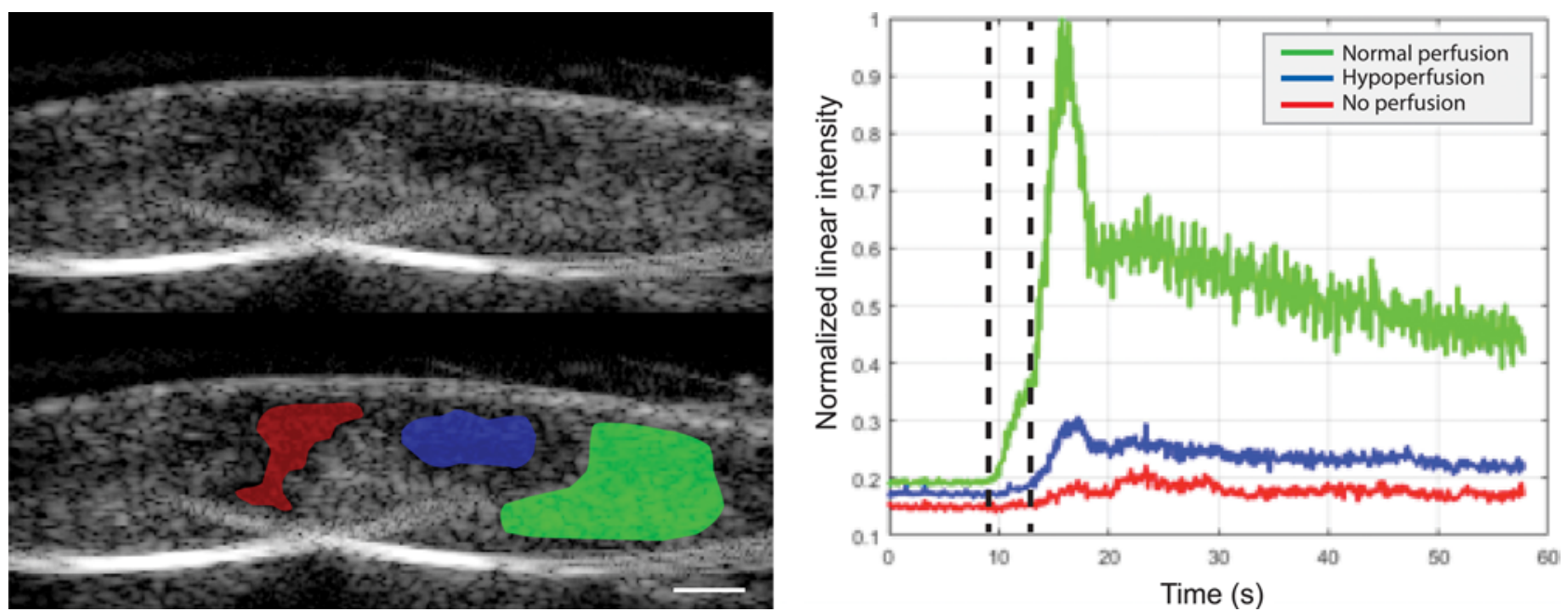

FIG. 3. Left: Sagittal CEUS image of an injured spinal cord with colors demarcating the injury center with a loss of perfusion (red), an area of hypoperfusion (blue), and an intact area (green). Right: Contrast inflow curves of the areas demarcated in the CEUS image.

days after acute SCI. ${ }^{39}$ However, current technologies do not allow for a real-time assessment of local spinal tissue perfusion.

Vascular events following tSCI have been studied utilizing terminal imaging techniques in animal tSCi models. ${ }^{34}$ Various angiographic methods followed by tissue histolo$\mathrm{gy}^{11,12,14}$ or by synchroton radiation microtomography have allowed visualization of spinal microvasculature. ${ }^{7}$ In vivo and intraoperative visualization of intrinsic spinal cord vasculature remains challenging. Routinely used clinical imaging modalities such as magnetic resonance angiography (MRA) and computed tomography angiography (CTA) do not provide adequate spatial resolution to allow for depiction of the spinal cord vasculature. Accordingly, in a recent clinical study utilizing CTA the limited imaging resolution allowed for visualization of only the largest spinal cord vessel (the anterior spinal artery) in patients with SCI. ${ }^{43}$ These in vivo imaging limitations have recently been overcome by the development of novel ultrasound imaging techniques.

Studies on SCI patients have utilized ultrasound to visualize post-traumatic spinal hematoma. ${ }^{4,93}$ In an animal model, intrinsic spinal vasculature has been visualized using high-frequency ultrasound systems combined with conventional Doppler. ${ }^{32}$ This study revealed vascular disruption and development of an expanding hematoma in the injury center. Similar to our findings, the authors detected destruction of microvasculature at the epicenter of the injury and subsequent mass effect onto the remaining adjacent vessels. Another study utilized intravenous contrast in combination with traditional ultrasound to study tissue perfusion kinetics following rodent tSCI. ${ }^{33}$ In this study, high-frequency CEUS revealed a dramatic loss of perfusion at the injury center of a T10 contusion injury. ${ }^{33}$ Importantly, the degree of hypoperfusion at the injury center and penumbra worsened during the 1st hour following trauma, indicating that ischemia might be preventable if treated early. However, unique to our set-up is the development of ultrafast plane-wave acquisitions, which allows capture of the passage of the microbubble bolus and separation of low-velocity blood flow within the microcirculation from higher-velocity flow in the macrocirculation. ${ }^{36-38}$ Thus, perfusion signal obtained using ultrafast CEUS Doppler is not contaminated by contrast signal from larger vessels. Our novel ultrafast CEUS Doppler allows, for the first time, assessment of spinal vasculature structural integrity.

Measurements of vessel diameter on CEUS were similar to measurements obtained in a histological analysis of spinal cord vessels. ${ }^{35}$ CEUS estimated the mean diam-

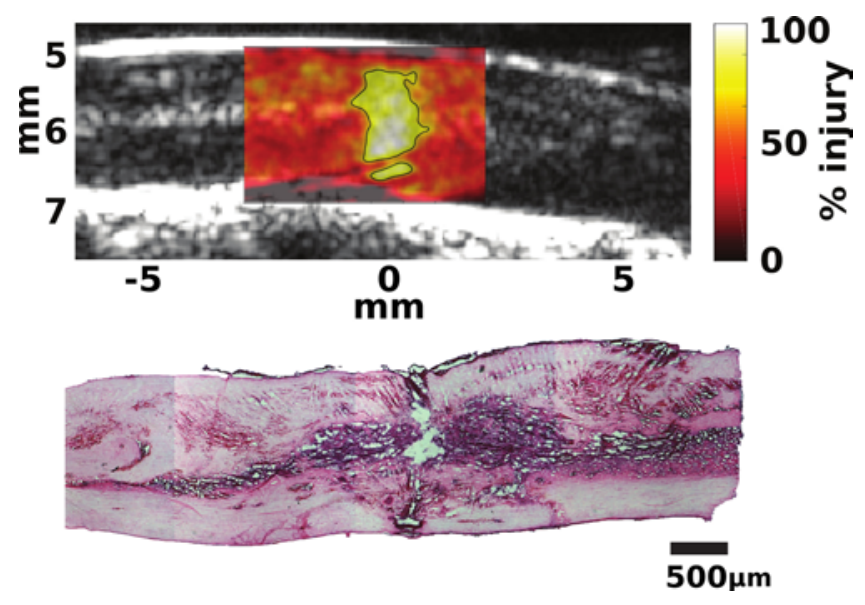

FIG. 4. Upper: Sagittal contour map delineating the injured spinal cord tissue with critically low tissue perfusion. The outlined area (yellow and white) demarcates the area of the injury with less than $40 \%$ tissue perfusion compared to surrounding healthy tissue. Lower: $\mathrm{H} \& \mathrm{E}$-stained sagittal section of the same spinal cord showing the acute $\mathrm{SCl}$. Note the close resemblance of the necrotic core to the area outlined in the sagittal contour map. 
eter of the anterior spinal artery as $145.8 \pm 10.0 \mu \mathrm{m}$ compared to the histological measurement of $110 \mu \mathrm{m}$ and the central arteries as $76.2 \pm 4.5 \mu \mathrm{m}$ compared to $51 \mu \mathrm{m} .{ }^{35}$ The slightly larger diameter obtained by CEUS measurements might have been caused by blooming of the high contrast levels within the blood vessels compared to surrounding tissue. Importantly, ultrafast CEUS Doppler provides a topographical map with high spatial resolution of the microcirculation that facilitates capillary exchange of oxygen, electrolytes, and nutrients. Laser Doppler, on the other hand, a technique widely used to assess spinal perfusion in various animal models of SCI, $, 18,19$ does not provide quantitative data regarding the extent of local hypoperfusion. Rather, laser Doppler provides a combined estimate of blood flow in a tissue volume under the probe to a depth of approximately $1-3 \mathrm{~mm}^{18}$ A topographical assessment of spinal cord perfusion in a rat in real time with the high spatial resolution provided by ultrafast CEUS Doppler has not been possible with other techniques.

\section{Clinical Significance}

Intraoperative ultrasound is in routine use in the neurosurgical operating room. It aids in the visualization of spinal compression or spinal tumors. In addition, CEUS is routinely used to visualize perfusion of the heart and liver around the world. Ultrasound contrast agents are also FDA-approved for imaging of the liver and heart in the US and have been in clinical use in the United States for over 20 years, with an excellent safety record. ${ }^{3,23}$ The technique proposed in the current report might, once translated into the clinic, be able to identify patients with a significant hypoperfused penumbra surrounding the center of the SCI. It might be hypothesized that such patients could be ideal candidates for neuroprotective interventions. Recently, decompression of the thecal sac has been shown to alleviate intraspinal pressure in rodents ${ }^{21}$ and humans. ${ }^{30}$ Furthermore, a recent clinical study demonstrated that both clinical motor response and amplitude of motor evoked potentials correlate with spinal perfusion pressure in patients with acute tSCI. ${ }^{40}$ Our current report suggests that CEUS could potentially help to detect and monitor local tissue perfusion at the injury site. We envision utilizing routine preoperative MRI for an estimation of the rostrocaudal extent of a spinal contusion ${ }^{1}$ in order to allow for complete intraoperative CEUS-based screening of the injury. Moreover, preoperative MRI could be useful to identify spared tissue that would serve as a reference for perfusion parameters of intact spared tissue. We also believe that intraoperative CEUS may be useful for additional indications within the field of spine surgery. CEUS-based monitoring of spinal cord perfusion could be a useful monitoring adjunct during reduction of severe spinal deformities or occlusion of spinal arteriovenous malformations. Moreover, intraoperative visualization of intrinsic spinal tumors using CEUS may help with lesion localization and confirmation of successful resection.

\section{Limitations}

The current study has several limitations. First, the calibrated compression method for tSCI results in a rela- tively small area of injury. Future studies are needed to describe loss of microvasculature and blood perfusion in a more common rodent SCI model (such as a contusion injury model). The topographical perfusion map obtained in more extensive injuries will then be compared to findings after human tSCI. Importantly, once translated to clinical use, ultrafast CEUS Doppler might help to better define the extremely heterogeneous population of human SCI patients. ${ }^{22}$ And vice versa, it will hopefully help to adjust and validate rodent animal models of tSCI, as they have been unreliable in predicting clinical treatment responses. Secondly, the proposed ultrafast CEUS Doppler can only be collected in patients who undergo direct posterior decompression of the injured spinal cord. Thus, patients who undergo anterior procedures or posterior stabilization-only procedures would not be candidates for this procedure. Moreover, while MRI does not provide the resolution to assess integrity of spinal blood vessels and local perfusion, recent advances in diffusion tensor imaging have suggested a potential role of this technique in the quantification of tract injury. ${ }^{20,29}$ Future studies are needed to compare diffusion tensor imaging with CEUS. Finally, in the patient population, time to surgery after tSCI is likely to be variable. In the current study, we examined the acute changes that occur after tSCI. However, future studies are needed to determine the usefulness of CEUS imaging at various time points after tSCI.

\section{Conclusions}

In the current study we present a novel ultrafast CEUS Doppler technique that allows for real-time imaging of low-velocity blood flow in the microcirculation and higher-velocity blood flow in the larger vasculature in a rodent tSCI. Once translated, this technique may be useful for intraoperative screening of structural and functional integrity of the spinal cord vasculature and its impact on functional outcome following tSCI.

\section{Acknowledgments}

This research was funded in part by a Royal Research Fund Grant (RRF-65-3210) and the Washington State Spinal Cord Injury Consortium (WASCIC).

\section{References}

1. Aarabi B, Simard JM, Kufera JA, Alexander M, Zacherl $\mathrm{KM}$, Mirvis SE, et al: Intramedullary lesion expansion on magnetic resonance imaging in patients with motor complete cervical spinal cord injury. J Neurosurg Spine 17:243-250, 2012

2. Abdullahi D, Annuar AA, Mohamad M, Aziz I, Sanusi J: Experimental spinal cord trauma: a review of mechanically induced spinal cord injury in rat models. Rev Neurosci 28:15-20, 2017

3. Aggeli C, Giannopoulos G, Roussakis G, Christoforatou E, Marinos G, Toli C, et al: Safety of myocardial flash-contrast echocardiography in combination with dobutamine stress testing for the detection of ischaemia in 5250 studies. Heart 94:1571-1577, 2008

4. Babyn PS, Chuang SH, Daneman A, Davidson GS: Sonographic evaluation of spinal cord birth trauma with pathologic correlation. AJR Am J Roentgenol 151:763-766, 1988 
5. Bartus K, James ND, Didangelos A, Bosch KD, Verhaagen J, Yáñez-Muñoz RJ, et al: Large-scale chondroitin sulfate proteoglycan digestion with chondroitinase gene therapy leads to reduced pathology and modulates macrophage phenotype following spinal cord contusion injury. J Neurosci 34:4822-4836, 2014

6. Cadotte DW, Fehlings MG: Spinal cord injury: a systematic review of current treatment options. Clin Orthop Relat Res 469:732-741, 2011

7. Cao Y, Wu T, Yuan Z, Li D, Ni S, Hu J, et al: Threedimensional imaging of microvasculature in the rat spinal cord following injury. Sci Rep 5:12643, 2015

8. Carlson GD, Gorden CD, Nakazawa S, Wada E, Smith JS, LaManna JC: Sustained spinal cord compression: part II: effect of methylprednisolone on regional blood flow and recovery of somatosensory evoked potentials. J Bone Joint Surg Am 85-A:95-101, 2003

9. de Vries E, Robben SG, van den Anker JN: Radiologic imaging of severe cervical spinal cord birth trauma. Eur J Pediatr 154:230-232, 1995

10. Ducker TB, Assenmacher DR: Microvascular response to experimental spinal cord trauma. Surg Forum 20:428-430, 1969

11. Ertürk A, Becker K, Jährling N, Mauch CP, Hojer CD, Egen JG, et al: Three-dimensional imaging of solvent-cleared organs using 3DISCO. Nat Protoc 7:1983-1995, 2012

12. Fairholm DJ, Turnbull IM: Microangiographic study of experimental spinal cord injuries. J Neurosurg 35:277-286, 1971

13. Fehlings MG, Vaccaro A, Wilson JR, Singh A, W Cadotte D, Harrop JS, et al: Early versus delayed decompression for traumatic cervical spinal cord injury: results of the Surgical Timing in Acute Spinal Cord Injury Study (STASCIS). PLoS One 7:e32037, 2012

14. Fried LC, Goodkin R: Microangiographic observations of the experimentally traumatized spinal cord. J Neurosurg 35:709-714, 1971

15. Furlan JC, Noonan V, Cadotte DW, Fehlings MG: Timing of decompressive surgery of spinal cord after traumatic spinal cord injury: an evidence-based examination of preclinical and clinical studies. J Neurotrauma 28:1371-1399, 2011

16. Guha A, Tator CH, Rochon J: Spinal cord blood flow and systemic blood pressure after experimental spinal cord injury in rats. Stroke 20:372-377, 1989

17. Hadley MN, Walters BC, Grabb PA, Oyesiku NM, Przybylski GJ, Resnick DK, et al: Guidelines for the management of acute cervical spine and spinal cord injuries. Clin Neurosurg 49:407-498, 2002

18. Hamamoto Y, Ogata T, Morino T, Hino M, Yamamoto H: Real-time direct measurement of spinal cord blood flow at the site of compression: relationship between blood flow recovery and motor deficiency in spinal cord injury. Spine (Phila Pa 1976) 32:1955-1962, 2007

19. Horn EM, Theodore N, Assina R, Spetzler RF, Sonntag VK, Preul MC: The effects of intrathecal hypotension on tissue perfusion and pathophysiological outcome after acute spinal cord injury. Neurosurg Focus 25(5):E12, 2008

20. Jirjis MB, Valdez C, Vedantam A, Schmit BD, Kurpad $\mathrm{SN}$ : Diffusion tensor imaging as a biomarker for assessing neuronal stem cell treatments affecting areas distal to the site of spinal cord injury. J Neurosurg Spine 26:243-251, 2017

21. Khaing ZZ, Cates LN, Fischedick AE, McClintic AM, Mourad PD, Hofstetter CP: Temporal and spatial evolution of raised intraspinal pressure following traumatic spinal cord injury. J Neurotrauma 34:645-651, 2017

22. Krishna V, Andrews H, Varma A, Mintzer J, Kindy MS, Guest J: Spinal cord injury: how can we improve the classification and quantification of its severity and prognosis? J Neurotrauma 31:215-227, 2014

23. Main ML: Contrast echocardiography: safety in numbers. Cardiology 122:248-250, 2012

24. Marshall LF, Smith RW, Shapiro HM: The outcome with aggressive treatment in severe head injuries. Part I: the significance of intracranial pressure monitoring. $\mathbf{J}$ Neurosurg 50:20-25, 1979

25. Mautes AE, Weinzierl MR, Donovan F, Noble LJ: Vascular events after spinal cord injury: contribution to secondary pathogenesis. Phys Ther 80:673-687, 2000

26. McDonough A, Monterrubio A, Ariza J, Martínez-Cerdeño $\mathrm{V}$ : Calibrated forceps model of spinal cord compression injury. J Vis $\operatorname{Exp}$ (98):52318, 2015

27. Miller JD, Butterworth JF, Gudeman SK, Faulkner JE, Choi SC, Selhorst JB, et al: Further experience in the management of severe head injury. J Neurosurg 54:289-299, 1981

28. Nimsky C, Carl B: Historical, current, and future intraoperative imaging modalities. Neurosurg Clin N Am 28:453-464, 2017

29. Peterson DJ, Rutman AM, Hippe DS, Jarvik JG, Chokshi FH, Reyes MR, et al: Test-retest and interreader reproducibility of semiautomated atlas-based analysis of diffusion tensor imaging data in acute cervical spine trauma in adult patients. AJNR Am J Neuroradiol 38:2015-2020, 2017

30. Phang I, Werndle MC, Saadoun S, Varsos G, Czosnyka M, Zoumprouli A, et al: Expansion duroplasty improves intraspinal pressure, spinal cord perfusion pressure, and vascular pressure reactivity index in patients with traumatic spinal cord injury: injured spinal cord pressure evaluation study. J Neurotrauma 32:865-874, 2015

31. Quencer RM: The injured spinal cord. Evaluation with magnetic resonance and intraoperative sonography. Radiol Clin North Am 26:1025-1045, 1988

32. Soubeyrand M, Badner A, Vawda R, Chung YS, Fehlings MG: Very high resolution ultrasound imaging for real-time quantitative visualization of vascular disruption after spinal cord injury. J Neurotrauma 31:1767-1775, 2014

33. Soubeyrand M, Laemmel E, Dubory A, Vicaut E, Court C, Duranteau J: Real-time and spatial quantification using contrast-enhanced ultrasonography of spinal cord perfusion during experimental spinal cord injury. Spine (Phila Pa 1976) 37:E1376-E1382, 2012

34. Tator $\mathrm{CH}$, Fehlings MG: Review of the secondary injury theory of acute spinal cord trauma with emphasis on vascular mechanisms. J Neurosurg 75:15-26, 1991

35. Tokioka T: The arterial system of the spinal cord in the rat. Okajimas Folia Anat Jpn 50:133-182, 1973

36. Tremblay-Darveau C, Williams R, Milot L, Bruce M, Burns PN: Combined perfusion and Doppler imaging using planewave nonlinear detection and microbubble contrast agents. IEEE Trans Ultrason Ferroelectr Freq Control 61:19882000, 2014

37. Tremblay-Darveau C, Williams R, Milot L, Bruce M, Burns PN: Visualizing the tumor microvasculature with a nonlinear plane-wave Doppler imaging scheme based on amplitude modulation. IEEE Trans Med Imaging 35:699-709, 2016

38. Tremblay-Darveau C, Williams R, Sheeran PS, Milot L, Bruce M, Burns PN: Concepts and tradeoffs in velocity estimation with plane-wave contrast-enhanced Doppler. IEEE Trans Ultrason Ferroelectr Freq Control 63:18901905,2016

39. Walters BC, Hadley MN, Hurlbert RJ, Aarabi B, Dhall SS, Gelb DE, et al: Guidelines for the management of acute cervical spine and spinal cord injuries: 2013 update. Neurosurgery 60 (Suppl 1):82-91, 2013

40. Werndle MC, Saadoun S, Phang I, Czosnyka M, Varsos GV, Czosnyka ZH, et al: Monitoring of spinal cord perfusion pressure in acute spinal cord injury: initial findings of the 
injured spinal cord pressure evaluation study. Crit Care Med 42:646-655, 2014

41. Wilson JR, Singh A, Craven C, Verrier MC, Drew B, Ahn $\mathrm{H}$, et al: Early versus late surgery for traumatic spinal cord injury: the results of a prospective Canadian cohort study. Spinal Cord 50:840-843, 2012

42. Wyndaele JJ: The impact of early versus late surgical decompression on neurological recovery after traumatic spinal cord injury (SCI). Spinal Cord 50:789, 2012

43. Zhang Z, Wang H, Zhou Y, Wang J: Computed tomographic angiography of anterior spinal artery in acute cervical spinal cord injury. Spinal Cord 51:442-447, 2013

\section{Disclosures}

The authors report no conflict of interest concerning the materials or methods used in this study or the findings specified in this paper.

\section{Author Contributions}

Conception and design: Hofstetter, Khaing, Mourad, Bruce. Acquisition of data: Khaing, Cates, DeWees, Hannah, Bruce. Analysis and interpretation of data: Hofstetter, Khaing, DeWees, Bruce. Drafting the article: Hofstetter, Khaing. Critically revising the article: Hofstetter. Reviewed submitted version of manuscript: Hofstetter. Approved the final version of the manuscript on behalf of all authors: Hofstetter. Statistical analysis: Hofstetter, Khaing, Hannah, Bruce. Administrative/technical/material support: Cates, DeWees, Hannah. Study supervision: Hofstetter, Mourad.

\section{Supplemental Information}

Videos

Video 1. https://vimeo.com/253958770.

Video 2. https://vimeo.com/253958779.

\section{Correspondence}

Christoph Hofstetter: The University of Washington, Seattle, WA. chh9045@uw.edu. 